\title{
Correlación entre la fiebre y la frecuencia respiratoria en menores de 5 años
}

\author{
CAROLINA GÓMEZ L. DE M. ${ }^{1}$, IVAN D. FLOREZ ${ }^{2}$, OLGA MORALES M. ${ }^{3}$, \\ MARCELA BERMÚDEZ C. ${ }^{4}$, JEISON AGUILAR H. ${ }^{4}$, LINA LÓPEZ V. ${ }^{5}$ \\ 1. Pediatra. Universidad de Antioquia. Colombia. \\ 2. Pediatra, Epidemiólogo clínico. Universidad de Antioquia. Colombia. \\ 3. Neumóloga Pediatra, Universidad de Antioquia. Colombia. \\ 4. Médico, Universidad de Antioquia. Colombia. \\ 5. Médico, residente medicina nuclear. Hospital Universitario Miguel Servet de Zaragoza, España.
}

\begin{abstract}
Correlation between fever and respiratory rate in children under 5 years of age

Introduction: Increased body temperature results in raised respiratory rate (RR) leading to tachypnea. According to World Health Organization (WHO), the diagnosis of pneumonia based on the presence of tachypnea can be overestimated during fever. The aim of this study is to determine the correlation between fever and RR in febrile children under five years of age. Patients and Method: A prospective study of correlation in children between 2 and 60 months was performed. RR and body temperature were measured on Emergency Room admission and after receiving acetaminophen. The correlation between the temperature change and the change in the RR was calculated. The frequency of tachypnea at admission and after normalizing the temperature was determined. Results: 362 patients were included, mean age 23.7 months old. RR in children under one year of age decreased 12.9 breaths per minute after normalizing the temperature, in older than one year of age, it decreased 10.7 breaths per minute. The $\mathrm{r}^{2}$ coefficient between temperature and $\mathrm{RR}$ was 0.08 for children under one year and 0.11 for older. Among the children who were tachypneic, $57 \%$ did not present this condition after normalizing the temperature. Conclusions: It is recommended to reevaluate RR in all febrile children after normalizing the temperature and before diagnosing tachypnea.
\end{abstract}

(Key words: Respiratory rate, tachypnea, fever, pneumonia).

Rev Chil Pediatr 2013; 84 (4): 409-416

\section{RESUMEN}

Introducción: Entre los efectos de la fiebre está el incremento de la frecuencia respiratoria (FR) hasta alcanzar rangos de generar taquipnea. El diagnóstico de neumonía según la OMS basado en la presencia de taquipnea puede sobreestimarse durante la fiebre. El objetivo de este estudio fue determinar la correlación entre fiebre

Recibido el 2 de marzo de 2012, devuelto para corregir el 04 de junio de 2012, segunda versión 20 de agosto de 2012, aceptado para publicación el 18 de enero de 2013.

Este trabajo cumple con los requisitos sobre consentimiento /asentimiento informado, comité de ética, financiamiento, estudios animales y sobre la ausencia de conflictos de intereses según corresponda.

Correspondencia a:

Carolina Gómez López de Mesa

E-mail: carogldm@hotmail.com 
y FR en niños febriles menores de 5 años. Pacientes y Método: Estudio prospectivo de correlación en niños entre 2 y 60 meses. Se midió FR y temperatura corporal al ingreso a Servicio de Urgencia y luego de recibir acetaminofén. Se calculó la correlación entre el cambio de temperatura y el cambio en la FR. Se determinó la frecuencia de taquipnea al ingreso y al normalizar la temperatura. Resultados: 362 pacientes fueron incluidos, media 23,7 meses (DE 16,1). La FR en menores de un año disminuyó 12,9 respiraciones por minuto (DE 6,7 ) al normalizar temperatura; en mayores, disminuyó 10,7 respiraciones por minuto (DE 5,2). El coeficiente $\mathrm{r}^{2}$ entre temperatura y FR fue 0,08 para menores de un año y 0,11 para mayores. De los niños que estaban taquipneicos, $57 \%$ no lo estaban al normalizar la temperatura. Conclusiones: Se recomienda reevaluar la FR de todo niño febril luego de normalizar su temperatura antes de definir taquipnea.

(Palabras clave: Frecuencia respiratoria, taquipnea, fiebre, neumonía).

Rev Chil Pediatr 2013; 84 (4): 409-416

\section{Introducción}

La fiebre es uno de los motivos de consulta más frecuentes en los servicios de urgencias pediátricas en todo el mundo, representando entre 20 y $40 \%$ de las consultas de los niños, principalmente en menores de 18 meses $^{1,2}$. Los procesos infecciosos causantes de fiebre son muy variables. Desde infecciones virales autolimitadas en la gran mayoría, hasta infecciones bacterianas como la neumonía, bacteriemia, meningitis e infecciones urinarias ${ }^{3}$, además de las causas no infecciosas. Entre estas últimas, cobra importancia la neumonía por ser la principal causa de muerte de niños menores de cinco años en todo el mundo ${ }^{4}$.

La medición de la frecuencia respiratoria (FR) es la principal herramienta para la identificación temprana de neumonía, puesto que al aumento en el valor normal para la edad (taquipnea) se le ha adjudicado una sensibilidad de $74 \%$ y especificidad de $67 \%{ }^{5}$. La Organización Mundial de la Salud (OMS), recomienda que todo niño menor de 5 años con tos o dificultad respiratoria y elevación de la FR para la edad, deba clasificarse como neumonía, independientemente de su temperatura corporal, y debe recibir tratamiento antibiótico ${ }^{6}$.

La fiebre ejerce un estimulo respiratorio que incrementa la FR y profundidad de la respiración ${ }^{7,8}$; por lo tanto, el conteo de la FR en niños durante el episodio febril, puede llevar a falsos positivos de neumonía ${ }^{9,10}$. Los resultados de los pocos trabajos publicados que han estudiado la correlación entre la fiebre y la FR son diversos ${ }^{9,11-13}$. O'Dempsey ${ }^{9}$, mostró un incremento de 3,7 respiraciones por minuto $(\mathrm{rpm})$ por cada grado centígrado $\left({ }^{\circ} \mathrm{C}\right)$. Gadomski ${ }^{11}$, por su parte, encontró aumento entre 5 y $7 \mathrm{rpm}$ para mayores de 1 año y entre 7 y $11 \mathrm{rpm}$ para menores de 1 año. Más recientemente, Davies ${ }^{13}$, encontró un incremento tan sólo de 0,5-2 rpm durante el episodio febril. Algunas de las limitaciones encontradas en los estudios mencionados fue la población, ya que la mayoría incluyó sólo menores de un año, a pesar de existir una alta prevalencia de cuadros febriles también en los niños mayores, mientras que otros se realizaron en adultos, cuyos resultados no son comparables con los de los niños, por razones fisiológicas y anatómicas. En ninguno de los estudios se evaluó si hubo una influencia de la temperatura ambiental sobre la FR.

El objetivo de este estudio es determinar la correlación existente entre la fiebre y la FR en los niños menores de 5 años.

\section{Pacientes y Método}

\section{Diseño del estudio}

Estudio descriptivo transversal, entre junio y diciembre de 2009, en el área de triage de urgencias pediátricas del Hospital Universitario San Vicente Fundación, y la Clínica SOMA en el municipio de Medellín, Antioquia (altitud: $1.475 \mathrm{msnm}$ ), el Hospital Francisco Valderrama del municipio de Turbo, Antioquia (altitud: 2 msnm) y el Hospital San Juan de Dios del municipio de Rionegro, Antioquia (altitud: $2.125 \mathrm{msnm})$. 


\section{Pacientes}

Mediante un muestreo no probabilístico de casos consecutivos se incluyeron todos los niños entre 2 y 60 meses de edad, que consultaron por fiebre de cualquier origen a los servicios de urgencias pediátricas de las instituciones participantes, independientemente del tiempo de inicio de ésta. Se excluyeron los pacientes con enfermedades graves (sepsis, dificultad respiratoria grave, deshidratación grave, meningitis, alteración del estado de conciencia), neumopatía crónica, ingesta de medicamentos que alteran la frecuencia cardíaca y/o la FR las $24 \mathrm{~h}$ previas (dextroanfetamina, fenilefrina, pseudoefedrina, etilefrina, fenilpropanolamina, metilfenidato, derivados del ergot o xantinas), ingesta de antipirético en las $2 \mathrm{~h}$ anteriores a la valoración, historia de reacción adversa o contraindicación al acetaminofen, y la presencia de estados dolorosos.

Se obtuvo el consentimiento escrito por parte del padre o tutor. Esta investigación fue aprobada por los comités de ética de las instituciones participantes.

\section{Mediciones}

Un equipo de salud conformado por 3 pediatras, uno de ellos neumólogo, 4 médicos de urgencias, 3 enfermeras y 2 estudiantes de medicina, previamente capacitados fueron los responsables de realizar a cada paciente la medición de la FR, la temperatura corporal y la temperatura ambiental, además de diligenciar el formulario del estudio y el formulario de atención.

La medición de la temperatura corporal se realizó mediante la ubicación de un termómetro de mercurio sobre la axila durante $3 \mathrm{~min}$ y la FR mediante la observación de los movimientos torácicos y abdominales que acompañaban a la respiración, durante un minuto, tal como lo recomienda la OMS. El paciente debía estar despierto, tranquilo, cómodo y sin llanto. Se definió fiebre como la temperatura corporal mayor o igual a $38^{\circ} \mathrm{C}$ tomada en la axila con un termómetro de mercurio. La temperatura ambiental fue obtenida con termómetros de pared que detectan temperaturas entre $-10^{\circ} \mathrm{C} \mathrm{y}+50^{\circ} \mathrm{C}$ por medio de una barra de mercurio líquido termo sensible. Luego de haber obtenido estas tres mediciones se administró una dosis oral de acetaminofén de $15 \mathrm{mg} / \mathrm{kg}$, y se esperó hasta el min 60 y 90 posterior a la administración del antipirético para realizar nuevamente las tres mediciones previamente descritas.

Se recolectó información sobre el motivo de consulta y el diagnóstico etiológico del niño. No se realizaron exámenes diagnósticos complementarios de forma rutinaria por protocolo en los pacientes, sólo fueron realizadas en los casos que el médico tratante lo consideró necesario. Se definió taquipnea según la edad, de acuerdo a las recomendaciones de la OMS: niños entre 2 y 11 meses, 50 o más rpm y niños mayores de un año y menores de cinco, 40 o más rpm.

\section{Análisis estadístico}

Se realizó un análisis descriptivo de las variables clínicas de los niños al ingreso. A las variables cuantitativas se les demostró distribución normal mediante la prueba de Kolmogorov-Smirnov. Definimos como variable independiente al cambio en la temperatura en grados Celsius y se obtuvo mediante la diferencia entre el valor al ingreso y el valor a los 90 min de cada uno de los niños; como variable dependiente al cambio en la FR obtenido a partir de la diferencia entre la FR al inicio y a los 90 min, de cada uno de los niños. En la búsqueda de la correlación entre estas variables se realizó para ambos grupos, menores y mayores de un año, un gráfico de dispersión y se calculó el coeficiente de correlación de Pearson, con base en los delta de cambio de FR y temperatura en cada niño. Se construyeron gráficos de dispersión entre las dos variables de los deltas de FR y temperatura de los dos grupos de edad y según el coeficiente de correlación obtenido, y se planteó la posibilidad de realizar un modelo de regresión lineal.

Los formularios de recolección de la información fueron digitados en una hoja de cálculo del programa Microsoft Office Excel 2007 y posteriormente llevados al software estadístico Stata versión 10,0 para su análisis estadístico. El tamaño de la muestra se calculó mediante el programa Epidat 3,1. Para un estudio de correlación, con un $\alpha$ : 0,05 , un $\beta: 0,2$ y un coeficiente de correlación de Pearson de 0,2, se obtiene 
como resultado una muestra de 153 pacientes. Para estratificarlo por dos grupos de edad: menores y mayores de un año, duplicamos la muestra obteniendo un total de 306 pacientes.

\section{Resultados}

Cumplieron con los criterios de inclusión 385 pacientes, sin embargo, de estos 7 no aceptaron participar en la investigación y en 16 no se realizó la firma del consentimiento informado. Finalmente, 362 pacientes entre 2 y 60 meses fueron incluidos en el estudio, con una media de 23,7 meses (DE 16,1). El 71,8\% (260) de los pacientes eran mayores de un año, el $54,1 \%$ (196) del sexo masculino y el $89,8 \%$ (325) vivían en zona urbana. La temperatura ambiental promedio fue de $31,6^{\circ} \mathrm{C}$ para Turbo, $25,9^{\circ} \mathrm{C}$ para Medellín y $16,9^{\circ} \mathrm{C}$ para Rionegro, con una altitud de $2,1.475$ y 2.125 metros sobre el nivel del mar (msnm), respectivamente (tabla 1). El reconocimiento de la fiebre en casa por la mayoría de los padres $(54,4 \%)$ fue de forma subjetiva, los restantes los hicieron mediante el uso del termómetro. La principal medida realizada por los padres para controlar la fiebre en casa fue la administración de acetaminofén $(296,81,8 \%)$, seguida por los medios físicos $(33,9,1 \%)$. No se realizó ninguna intervención en casa en 19 niños $(5,3 \%)$. Otros antipiréticos suministrados fueron antiinflamatorios no esteroidales (AINES) $(3,3 \%)$ y acido acetil salicílico $(0,6 \%)$. La etiología de la fiebre varió de acuerdo al grupo etario; en los menores de 1 año las 5 principales causas de fiebre encontradas fueron infección urinaria $22,6 \%$ (23 niños), infección respiratoria aguda $21,6 \%$ (22 niños), enfermedad diarreica aguda $21,6 \%$ (22 niños), neumonía 19,6\% (20 niños) y bronquiolitis $8,8 \%$ (9 niños), mientras que para los mayores de 1 año fueron infección respiratoria aguda $28,5 \%$ (74 niños), enfermedad diarreica aguda $16,9 \%$ (44 niños), otitis media aguda 11,2\% (29 niños), infección urinaria $9,6 \%$ (25 niños) y neumonía $8,1 \%$ (21 niños). En los menores de un año la media de la FR fue de 56,5 rpm (DE 11,35) al ingreso; a los 60 y 90 min de administrado el acetaminofén fue de 48,9 rpm (DE 10,4) y 43,5 rpm (DE 10,30), respectivamente. En los mayores de un año fue de 43,28 rpm (DE 10,21) al ingreso, de 36,08 rpm (DE 9,38) al min 60 y 32,59 (DE 8,95) al min 90 . La media de la temperatura al ingreso fue $38,7^{\circ} \mathrm{C}$ tanto en los mayores como en los menores de 1 año. En la tabla 2 se detallan los valores de temperatura y FR en los tres momentos. En la tabla 3 se muestra el cambio en la FR y en la temperatura calculado como la diferencia entre el minuto cero y el min 90. El cambio en la temperatura desde el ingreso hasta el min 90 fue $1,66{ }^{\circ} \mathrm{C}$ en menores de un año, y de $1,67^{\circ} \mathrm{C}$ en los mayores de un año. El cambio en la FR desde el ingreso (niño febril) hasta el min 90 (niño afebril) fue de 12,95 rpm, DE 6,69 (1-37) en los menores de un año y de 10,69 rpm DE 5,23 (1-26) en los mayores de 1 año (tabla 3). De los menores de un año, 72 $(70,6 \%)$ estaban taquipneicos al ingreso, mientras que al desaparecer la fiebre, en el min 90,

Tabla 1. Característica sociodemográficas de los pacientes

\begin{tabular}{|lcccc|}
\hline & Todos & Medellín & Turbo & Rionegro \\
\hline n (\%) & $362(100)$ & $203(56)$ & $109(30,1)$ & $50(13,8)$ \\
\hline Edad en meses, media (rango) & $23,7(2-60)$ & $24,2(2-60)$ & $23,7(2-58)$ & $22,38(2-60)$ \\
\hline Menores de 1 año, n (\%) & $102(28,1)$ & $57(28)$ & $29(26,6)$ & $16(32)$ \\
Masculino, n (\%) & $196(54,1)$ & $112(55,1)$ & $61(55,9)$ & $23(46)$ \\
\hline Zona urbana, n (\%) & $325(89,7)$ & $199(98,1)$ & $78(71,5)$ & $48(96)$ \\
Temperatura corporal al ingreso & 38,76 & 38,73 & 38,81 & 38,78 \\
' $\mathrm{C}$, IC 95 & $(38,0-40,4)$ & $(38,0-40,3)$ & $(38,0-40,4)$ & $(38,0-39,8)$ \\
\hline $\begin{array}{l}\text { Temperatura ambiental media, } \\
{ }^{\circ} \mathrm{C}, \text { IC 95\% }\end{array}$ & 26,30 & 25,9 & 31,6 & 16,9 \\
\hline
\end{tabular}


Tabla 2. Cambio en la FR y la temperatura corporal a los 60 y 90 min de administrado el antipirético para menores y mayores de 1 año

\begin{tabular}{|lll|}
\hline & Menores de 1 año & Mayores de 1 año \\
\hline Min $\mathbf{0}$ & $\mathrm{n}=102$ & $\mathrm{n}=260$ \\
Media ${ }^{\circ} \mathrm{C}, \mathrm{DE},(\mathrm{IC} \mathrm{95 \% )}$ & $38,7,0,47(38,0-39,9)$ & $38,7,0,52(38,0-40,4)$ \\
Media FR, DE, rango & $56,5,11,35(32-87 \mathrm{rpm})$ & $43,2,10,21(22-86)$ \\
Min $\mathbf{6 0}$ & & \\
Media, ${ }^{\circ} \mathrm{C}, \mathrm{DE}$, (IC 95\%) & $37,61,0,51(36,0-38,7)$ & $37,7,0,50\left(36,1-39,33^{\circ} \mathrm{C}\right)$ \\
Media FR, DE, rango & $48,9,10,43(31-76)$ & $36,08,9,38(16-78)$ \\
Min 90 & & \\
Media, ${ }^{\circ} \mathrm{C}, \mathrm{DE},(\mathrm{IC}$ 95\%) & $37,04,1,13(36,6-38,0)$ & $37,11,0,43(36,0-38,5)$ \\
Media FR, DE, rango & $43,54,10,30(23-70)$ & $32,59,8,95(18-80)$ \\
\hline
\end{tabular}

Minuto 0: antes de suministrar antipirético, min 60: 60 minutos luego de administrado el antipirético, min 90: 90 minutos luego de administrado el antipirético, FR: Frecuencia respiratoria en un min. ${ }^{\circ} \mathrm{C}$ : grados celcius.

Tabla 3. Cambio en la FR y la temperatura corporal desde el ingreso (minuto 0) hasta el min 90 y proporción de niños con taquipnea en los dos momentos

\begin{tabular}{|lrr|}
\hline & $<\mathbf{1}$ año & $>1$ año \\
\hline Cambio en la FR (rpm), DE & $-12,95(6,69)$ & $-10,69(5,23)$ \\
Cambio en la temperatura ${ }^{\circ} \mathrm{C}, \mathrm{DE}$ & $-1,66(1,11)$ & $-1,67 \quad(0,54)$ \\
Niños taquipneicos febriles al ingreso, $\mathrm{n}(\%)$ & $72(70,59)$ & $168(64,62)$ \\
Niños taquipneicos afebriles al minuto $90, \mathrm{n}(\%)$ & $31(30,39)$ & $55(21,15)$ \\
\hline
\end{tabular}

FR: frecuencia respiratoria.

sólo 31 de ellos $(30,4 \%)$ lo estaban. De la misma forma, en mayores de un año, hubo descenso de la FR al desaparecer la fiebre, donde 168 $(64,6 \%)$ se encontraron taquipneicos con la fiebre al ingreso y al min 90, sólo 55 (21,2\%) continuaban taquipneicos.

Se realizó la prueba de correlación de Pearson entre el cambio de la temperatura y el cambio de la FR entre los minutos 0 y 90 y se

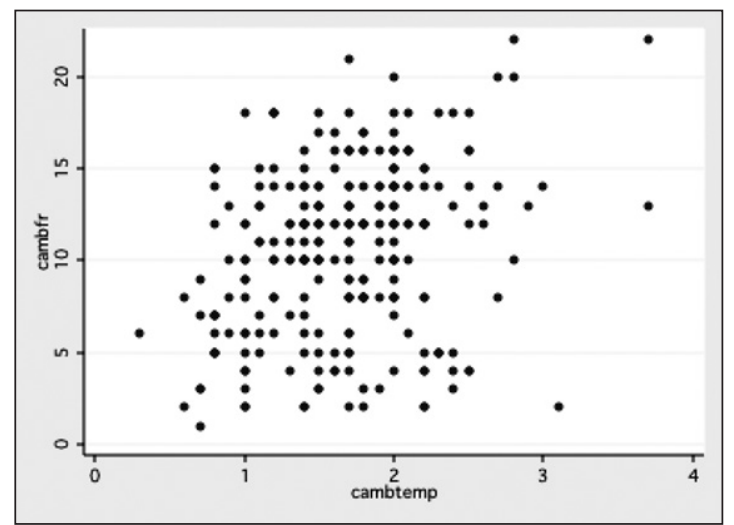

Figura 1. Gráfico de dispersión del cambio en la frecuencia respiratoria $(\mathrm{rpm})$ y el cambio en la temperatura $\left({ }^{\circ} \mathrm{C}\right)$, en mayores de un año, $r=0,33$. encontró un coeficiente de correlación $(r)$ de 0,33 para los mayores de un año y 0,29 para los menores. El gráfico de dispersión de puntos para ambos grupos de edad se muestra en la figuras 1 y 2 , los cuales a pesar de que muestran que a mayor cambio en la temperatura corporal, mayor cambio en la FR, pero con hallazgos bastante variables.

Es evidente tanto en la gráfica como en

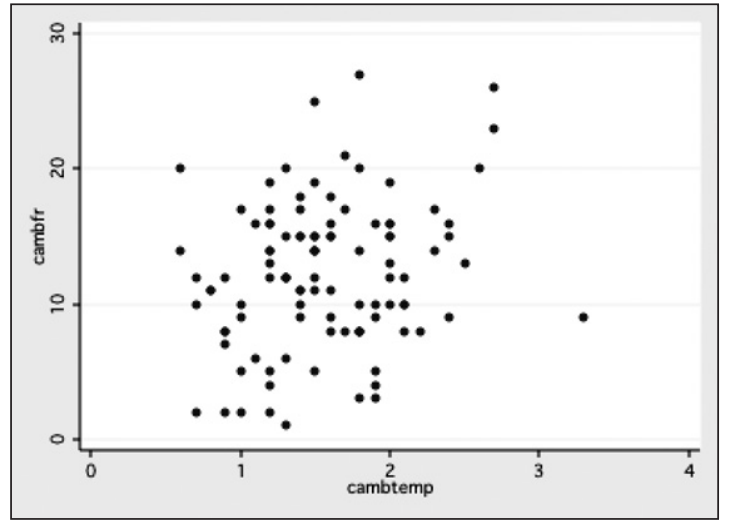

Figura 2. Gráfico de dispersión del cambio en la frecuencia respiratoria (rpm) y el cambio en la temperatura $\left({ }^{\circ} \mathrm{C}\right)$, en menores de un año, $r=0,29$. 
el valor de $r$ que la correlación es baja. Esto imposibilitó la construcción de un modelo de regresión lineal. No se encontró un cambio significativo de la FR al analizarlo por las diferentes altitudes sobre el nivel del mar.

\section{Discusión}

La fiebre es y seguirá siendo un importante motivo de consulta en los servicios de urgencias pediátricas, considerada frecuentemente como una enfermedad por sí misma más que como un síntoma ${ }^{14,15}$. La fiebre subjetiva detectada por los padres mediante palpación ha sido cuestionada, pero esta tiene reconocida validez y en nuestra población fue la más importante forma de detección del cuadro febril $(54,4 \%)$. Guías internacionales aceptadas recomiendan la percepción de la fiebre por los padres como un método válido y confiable debido a la buena correlación entre esta percepción y el valor objetivo de la temperatura corporal ${ }^{2,16}$. Respecto al uso de antipiréticos por parte de los padres, se ha encontrado que frecuentemente es incorrecto, tanto en dosis como en frecuencia ${ }^{17}$; en nuestro estudio el más ampliamente usado en casa fue el acetaminofén (81,7\%). Elegimos en nuestro estudio el uso de acetaminofén como antipirético por su perfil de seguridad.

La neumonía es la principal causa de muerte de niños entre un mes y cinco años de edad en todo el mundo y por ello es objetivo fundamental de los programas y estrategias de la OMS el promover su diagnóstico y tratamiento precoz. Es claro, que la misma OMS, en la búsqueda de herramientas para la detección precoz de esta infección ha logrado simplificar el abordaje con el conteo de la FR. De este modo, el niño que tenga taquipnea debe ser clasificado como portador de neumonía y excluyendo las sibilancias, prácticamente este se ha convertido en el parámetro más importante recomendado por este organismo para la clasificación de neumonía. Esto ha recibido variadas críticas puesto que la taquipnea como parámetro aislado presenta falsos positivos. Incluso algunas publicaciones han demostrado la baja especificidad de la taquipnea para detección de neumonía ${ }^{18-20}$. A este problema se le adiciona además, el ya descrito efecto que tie- ne la fiebre sobre la FR, constituyendo uno de los más grandes factores implicados en estos falsos positivos.

Buscamos la relación de la FR con varios factores, enfatizando en la temperatura corporal, edad, temperatura ambiental y altitud sobre el nivel del mar. Así mismo, quisimos demostrar una correlación al menos aceptable, entre el cambio en la FR y el cambio en la temperatura, con el fin de realizar un análisis de regresión lineal, buscando generar un modelo que tuviera un poder de predicción, el cual mostró baja correlación, sugiriendo que el cambio en la FR no es explicado solamente por el cambio en la temperatura. Factores como la ansiedad del niño, de la madre o padre, pueden estar asociados.

Este estudio muestra que no se puede proponer con exactitud un número absoluto de rpm, debido a que la FR es influenciada por múltiples factores, especialmente en los menores de un año ${ }^{13}$. En el momento de la consulta a urgencias, es muy frecuente la taquipnea en el niño febril, independiente de la causa etiológica. Este estudio reporta que un porcentaje significativo de pacientes dejaron de estar taquipneicos luego de la normalización de la temperatura: en los menores de un año de edad de aquellos que tenían taquipnea al ingreso, menos de la mitad continuaban con ella al min 90. En los mayores de un año la disminución en la proporción de taquipnea fue incluso, mucho mayor. Es decir, aunque hay variabilidad en el grado de disminución, es clara la disminución entre los momentos inicial y los $90 \mathrm{~min}$, pero ella no puede ser predecida muy probablemente por la existencia de abundantes factores relacionados con el control respiratorio bien sea orgánicos o incluso, del orden mental, las cuales desconocemos o simplemente no podemos identificar ni cuantificar. En este trabajo excluimos aquellos niños que al ingreso presentaban dificultad respiratoria, o ameritaban suplementación de oxígeno o de inhaloterapia, lo cuál sugiere que los hallazgos son reflejo de los cambios en la FR en niños que no tienen desde el ingreso un cuadro respiratorio importante y sintomático y pueden ser extrapolables a cualquier enfermedad que se asocie con fiebre. 
Con base en esto, debe considerarse siempre que todo niño febril tendrá una FR falsamente elevada y se hace necesario reevaluarla a la luz de un estado de normotermia luego de la administración del antipirético escogido. La adopción de la recomendación de la OMS de considerar a la taquipnea como signo único aislado de neumonía no grave independiente de la temperatura que posea el niño de forma aislada traerá consigo falsos dignósticos de neumonía y por lo tanto, uso irracional de antibióticos y con ello, predisposición al continuo incremento en la resistencia bacteriana en la comunidad.

Recomendamos que en la evolución de todo niño febril, si este presenta taquipnea con base en los puntos de corte recomendados por la OMS, debe realizarse una nueva medición de la FR posterior a la administración del antipirético, antes de tomar decisiones como el inicio de antibióticos, mucho más si se conoce que los niños con taquipnea tienen mayor probabilidad de tener radiografías que evidencien neumonía que los que no la presentan ${ }^{18}$.

Este estudio tiene limitaciones. Primero, nos basamos exclusivamente en los puntos de corte recomendados por la OMS: uno para los menores de dos meses (60 rpm), otro para los menores de un año y mayores de dos meses (50 rpm) y otro, para los mayores de un año (40 rpm). Estos puntos de corte son una excelente ayuda para el personal que atiende niños en un servicio de salud en un primer nivel por que de una forma global permiten diferenciar los niños taquipneicos de aquellos que no lo estan, pero como cualquier punto de corte esto no es absoluto y por tanto niños con FR por encima de ellos, pueden ser normales y otros con FR por debajo de ellos, pueden estar taquipneicos. Además, la imprecisión de dichos puntos se debe en gran parte a que agrupan niños que pueden ser sustancialmente distintos entre si como los menores de un año, grupo en el cuál se encuentran lactantes menores (2 ó 3 meses de edad) y niños de 10 a 11 meses. Este grupo resultará amplio y su desarrollo neurológico, cardiovascular y respiratorio será sustancialmente distinto entre ellos. Aunque es probable que sea mejor usar puntos de corte con grupos más estrechos y parecidos entre si, las recomendaciones de la OMS tienen ante todo, la cualidad de ser muy prácticas y de fácil aprendizaje, lo cuál les permite su aplicación en cualquier servicio y por cualquier profesional de salud. Segundo, dado que el objetivo era evaluar la relación entre FR y temperatura, no se buscó activamente el diagnóstico de neumonía y su relación con la taquipnea, como si lo han hecho otros trabajos ${ }^{18-20}$, imposibilitándonos de evaluar taquipnea como factor de predicción o "prueba diagnóstica" de neumonía. Sólo podemos mencionar que la disminución de la temperatura se relaciona con disminución de la FR y por lo tanto, con un menor número de niños considerados taquipneicos. Por último, a pesar de que intentamos ser uniformes en las condiciones de la toma de la FR, teniendo en cuenta la variabilidad encontrada en el cambio de ella, consideramos que nuestra población tuvo demasiados factores relacionados con la FR. Tal vez la exclusión de algunos pacientes que demostraran ansiedad al ingreso o la escogencia de niños sólo en estado de vigilia o mientras dormían, hubiese permitido delimitar mejor la población, disminuir la variabilidad y por lo tanto, mejorar la correlación.

En conclusión, se recomienda reevaluar la FR de todo niño febril luego de normalizar su temperatura antes de definir taquipnea.

\section{Referencias}

1.- Stewart M, Werneke U, MacFaul R, Taylor-Meek, Smith $H E$, Smith IJ: Medical and social factors associated with the admission and discharge of acutely ill children. Arch Dis Child 1998; 79 (3): 219-24.

2.- Richardson M, Lakhanpaul M: Assessment and initial management of feverish illness in children younger than 5 years: summary of NICE guidance. BMJ 2007; 334 (7604): 1163-4.

3.- Ishimine P: Fever without source in children 0 to 36 months of age. Pediatr Clin North Am 2006; 53 (2): 167-94.

4.- Black R, Morris SS, Bryce J: Where and why are 10 million children dying every year? Lancet 2003; 361: 2226-34.

5.- Morley C: Diagnostic value of tachypnoea. Arch Dis Child 2000; 82 (6): 503-5.

6.- World Health Organisation: Acute respiratory infections in children: case management in small hospitals in de- 
veloping countries. (WHO/ARI/90.5.) Geneva: WHO, 1990.

7.- Mackowiak PA, Worden G: Carl Reinhold August Wunderlich and the evolution of clinical thermometry. Clin Infect Dis 1994; 18 (3): 458-67.

8.- Caruana B, Gleeson K, Zwillich CW: The control of breathing in clinical practice. Chest 2000; 117 (1): 205-25.

9.- O'Dempsey TJD, Laurence BE, McArdle TF, Todd JE, Lamont AC, Greenwood BM: The effect of temperature reduction on respiratory rate in febrile illnesses. Arch Dis Child 1993; 68: 492-5.

10.- Campell H, Byass P, O'Dempsey TJ: Effects of body temperature on respiratory rate in young children. Arch Dis Child 1992; 67: 664-8.

11.- Gadomski A, Permutt T, Stanton B: Correcting respiratory rate for the presence of fever. J Clin Epidemiol 1994; 47 (9): 1043-9.

12.- Iliff A, Lee VA: Pulse rate, respiratory rate, and body temperature of children between two months and eighteen years of age. Child Dev 1952; 23 (4): 237-45.

13.- Davies P, Maconochue I: The relationship between body temperature, heart rate and respiratory rate in children.
Emerg Med J 2009; 26: 641-3.

14.- El-Radhi AS: Why is the evidence not affecting the practice of fever management?. Arch Dis Child 2008; 93: 918-20.

15.- Schmitt BD: Fever phobia: misconceptions of parents about fevers. Am J Dis Child 1980; 134 (2): 176-81.

16.- Walsh A, Edwards H, Fraser J: Attitudes and subjective norms: determinants of parents' intentions to reduce childhood fever with medications. Health Educ Res 2009; 24 (3): 531-45.

17.- Goldman RD, Ko K, Linett LJ, Scolnik D: Antipyretic efficacy and safety of ibuprofen and acetaminophen in children. Ann Pharmacother 2004; 38 (1): 146-50.

18.- Shah S, Bachur R, Kim D, Neuman MI: Lack of Predictive Value of Tachypnea in the Diagnosis of Pneumonia in Children. Pediatr Infect Dis J 2010; 29: 406-9.

19.- Nascimento CM, Benguigui Y: Evaluation of the degree of tachypnea for hospitalizing children with pneumonia. Indian Pediatr 2004; 41 (2): 175-9.

20.- King EA, Slawson DC: Tachypnea as a predictor of pneumonia in febrile children. J Fam Pract 1996; 42 (1): 25-9. 